\title{
Pleurectomy for spontaneous pneumothorax in cystic fibrosis
}

\author{
PETER F. MITCHELL-HEGGS and JOHN C. BATTEN \\ Brompton Hospital, London, S.W.3
}

\begin{abstract}
Five cases of cystic fibrosis complicated by eight pneumothoraces are described. These cases come from a group of 49 patients who have attended the Brompton Hospital between 1964 and 1969 for management of their cystic fibrosis. Three of the patients, who had a total of six pneumothoraces, were managed by pleurectomy. Spontaneous pneumothoraces were a terminal event in a fourth patient, and an incidental finding which required no specific management in a fifth patient. The possible aetiology of the pneumothorax in cystic fibrosis is discussed with particular consideration of the known alveolar hypoplasia and overdistension and the abnormality of the visceral pleura. The results of pleurectomy in three patients are discussed both in relation to the successful avoidance of further pneumothoraces and in the notable absence of a restrictive ventilatory defect. In view of the relative success of pleurectomy we suggest that this treatment should be considered for any patient with cystic fibrosis who develops a spontaneous pneumothorax.
\end{abstract}

Spontaneous pneumothorax is a recognized complication of cystic fibrosis (Kulczycki and LoPresti, 1967), but the actual incidence is not known; di Sant 'Agnese and Andersen (1959) discussed three patients with this complication in a summary of their series of 550. A recent report (Lifschitz, Bowman, Denning, and Wylie, 1968) suggests an incidence of $2.8 \%, 20$ cases occurring in 710 patients seen in a 15-year period. Eight pneumothoraces occurred in our five patients; three patients (two female and one male) with a total of six pneumothoraces were managed by pleurectomy and their management is discussed in detail. Two further cases, both males, are also mentioned.

\section{MATERIAL}

The three patients reported here are from a group of 49 with cystic fibrosis whose ages ranged from 5 to 33 years, with a mean of 22 years; they were seen at the Brompton Hospital between 1964 and 1969. There were 26 males and 23 females. Amongst other routine investigations regular measurements were made of forced expiratory volume in one second $\left(F E V_{1.0}\right)$ and forced vital capacity (FVC) using a Vitalograph; chest radiographs were also taken. Results of respiratory function are not available after pleurectomy in case 3. A fourth patient who died at the age of 16 years had terminal pneumothoraces, and in a fifth patient a left pneumothorax was noted as an incidental finding which resolved spontaneously.

\section{CASE HISTORIES}

CASE 1 J.W. D.O.B. 21.12.37. Cystic fibrosis was diagnosed at the age of 14 years. Sweat sodium concentration was $150 \mathrm{mEq} / \mathrm{l}$.; this fell to $106 \mathrm{mEq} / 1$. after she received 9 alpha fluoro-hydrocortisone, $18 \mathrm{mg}$. daily $(0.3 \mathrm{mg} . / \mathrm{kg}$.) for four days. Duodenal aspiration showed that tryptic activity was present in a greater than 1:10 concentration. On a fat intake of 70 g. per day there was a $66 \%$ absorption. One sister died aged 20 years from cystic fibrosis, complicated terminally by pneumothoraces.

Respiratory function gradually deteriorated from 1965. The patient developed a left pneumothorax at the end of April 1966 which was treated with an intercostal tube. A pneumothorax was induced successfully on 16.6.66 (Fig. 1). On 22.6.66 the left parietal pleura was stripped from apex to hilum and down the aorta to the diaphragm under general anaesthesia. Post-operative recovery was uneventful. The patient remained fairly well until October 1968. There was, however, a gradual increase in purulent sputum and dyspnoea, although she had had no time off work. In December she was found to have a shallow right pneumothorax which was managed conservatively with gradual resolution of the pneumothorax.

CASE 2 L.H. D.O.B. 26.6.52. Cystic fibrosis was diagnosed at the age of 6 years. Malabsorption and steatorrhoea were noted as well as a sweat sodium concentration of $144 \mathrm{mEq} / 1$. One sister died at the age of 6 months from 'pneumonia' of unknown cause. 


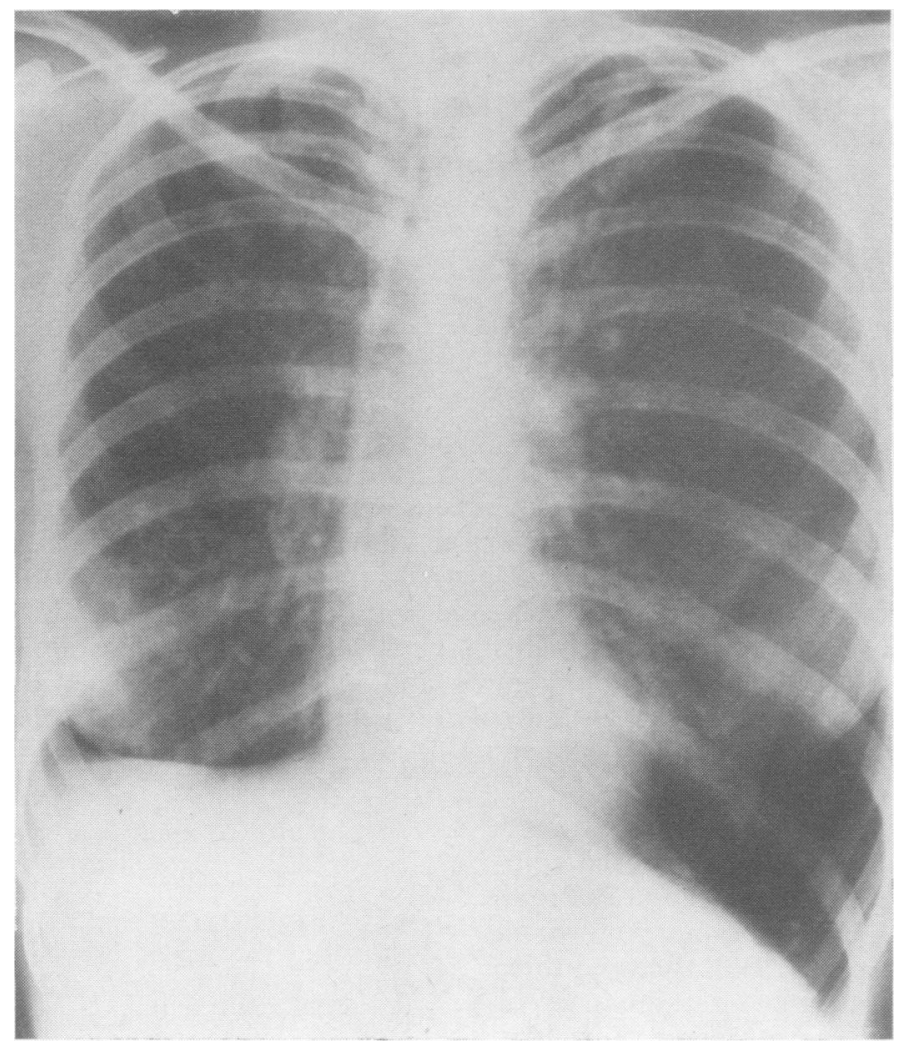

FIG. 1. Case 1. Chest radiograph (17.6.66) showing left pneumothorax.

T A B L E

FEV $_{1.0}$ AND FVC BEFORE AND AFTER PLEURECTOMY IN CASES 1 AND 2

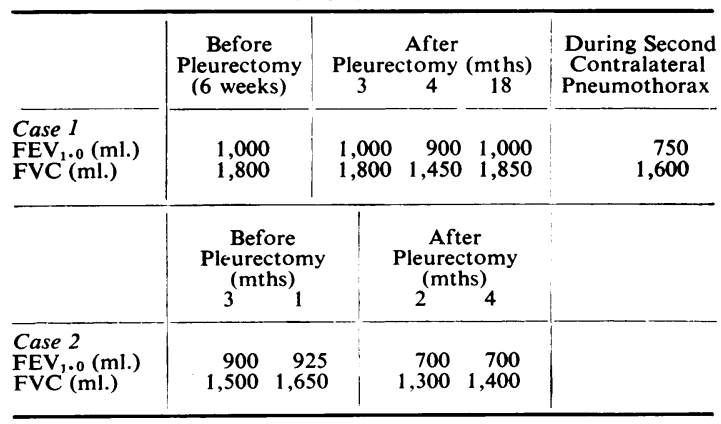

The patient had a moderate cough with mucoid and only occasionally purulent sputum which had developed at the age of 8 years. This continued without much change until early 1968 ; she then became breathless after an acute upper respiratory tract infection. There was a sudden onset of breathlessness and right-sided chest pain in June 1968, when the chest radiograph showed bilateral pneumothoraces; there was no mediastinal shift. She was initially managed conservatively and the right and left lungs expanded well. Three weeks later, however, there was a further collapse of the left lung (Fig. 2) and on 22.7.68 the left parietal pleura was stripped from the apex to the hilum and to the pericardium in front, backwards to the aorta and downwards to the diaphragm. Post-operative recovery was satisfactory and her clinical condition has remained unchanged (Fig. 3).

CASE 3 J.B. D.O.B. 23.5.54. Cystic fibrosis was diagnosed at the age of $12 \frac{1}{2}$ years. He had had purulent sputum since the age of 10 . One sister died young and had been 'prone to coughs and colds'.

The patient had become worse since 1966. There were no bowel symptoms. The sweat sodium concentration, $108 \mathrm{mEq} / 1$., was depressed to $96 \mathrm{mEq} / 1$. after receiving 9 alpha fluoro-hydrocortisone, $10 \mathrm{mg}$. daily for three days. The faecal fat was $3.3 \mathrm{~g}$./day. The chest radiograph appearances at the time are shown in Figure 4.

The patient developed a right pneumothorax on 1.5.68. This was managed with an intercostal tube in 


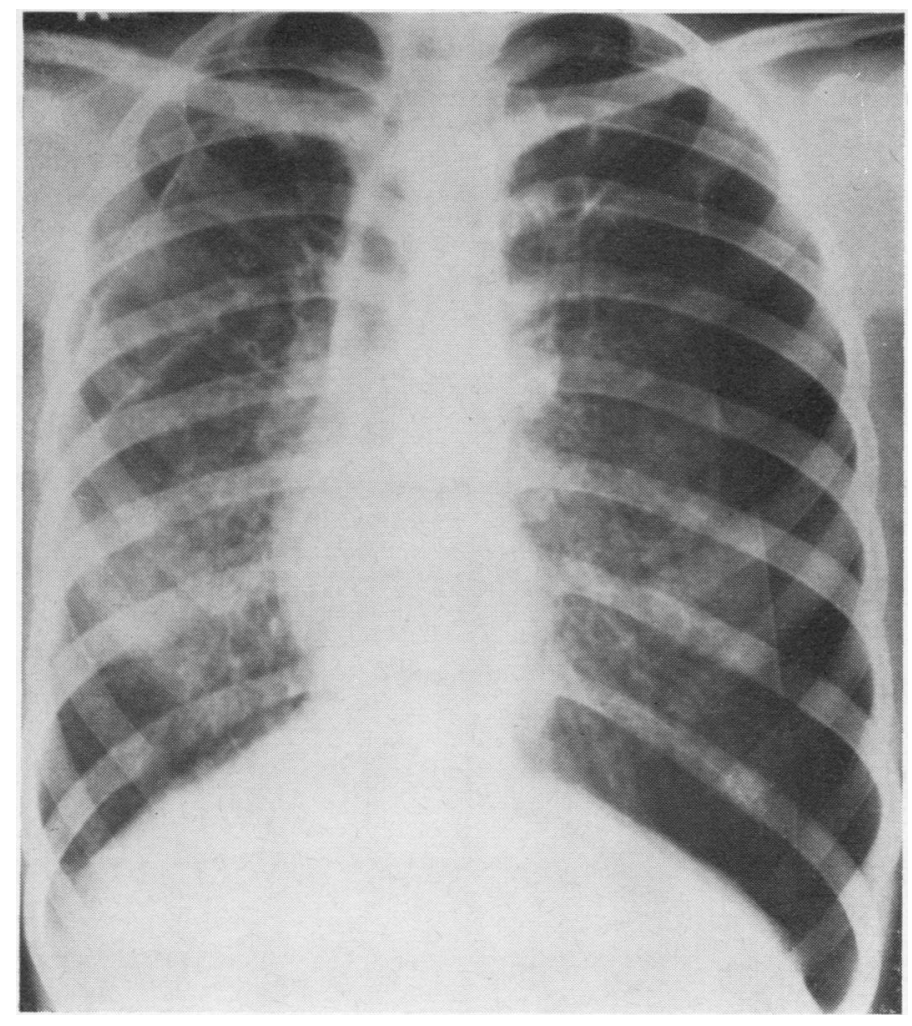

FIG. 2. Case 2. Chest radiograph (19.7.68) showing (second) left-sided pneumothorax.

FIG. 3. Case 2. Chest radiograph (16.12.68) nearly five months after surgery showing complete ablation of pneumothorax cavities.

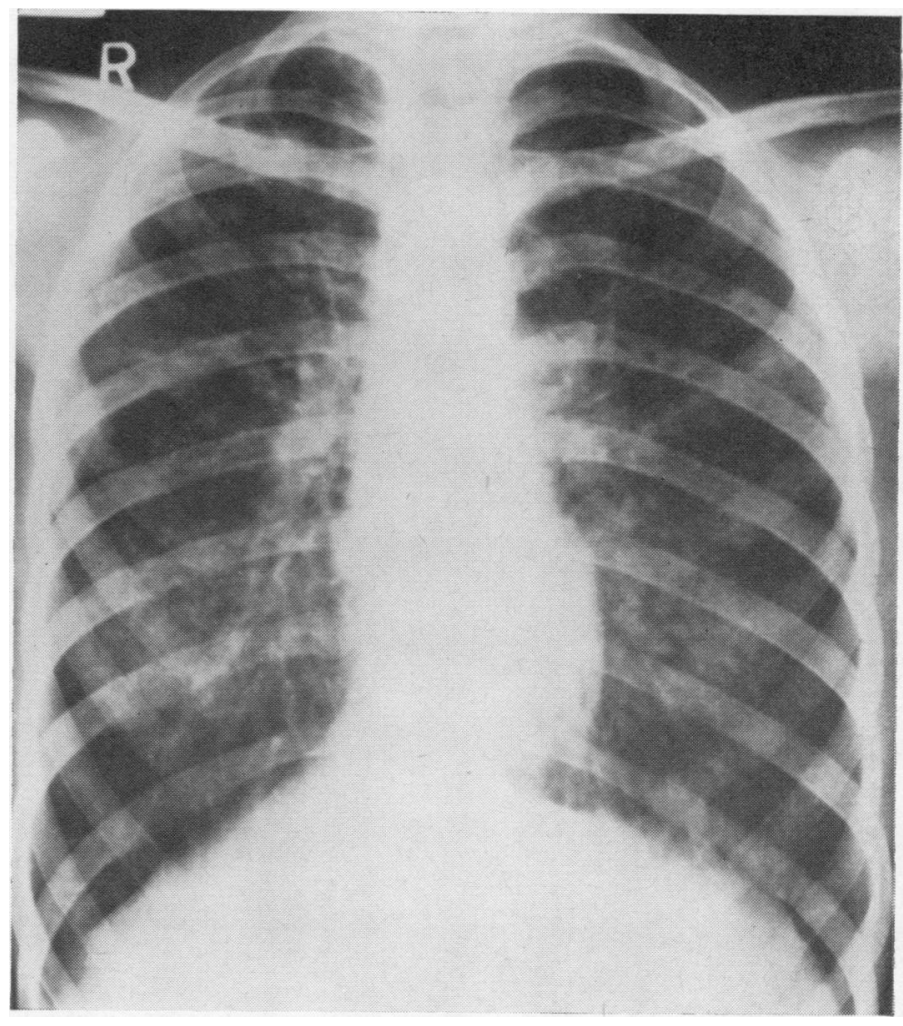




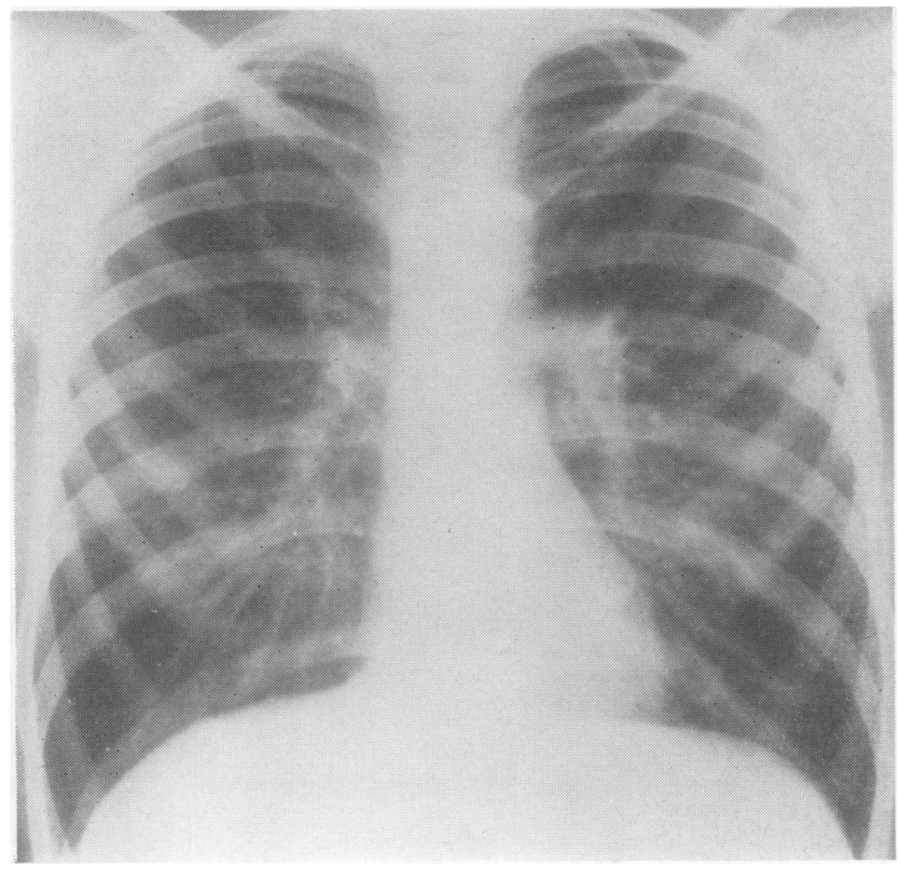

FIG. 4. Case 3. Chest radiograph (5.4.67) showing appearances in the lung fields typical of cystic fibrosis.

FIG. 5. Case 3. Chest radiograph (10.5.68) showing recurrent right pneumothorax.

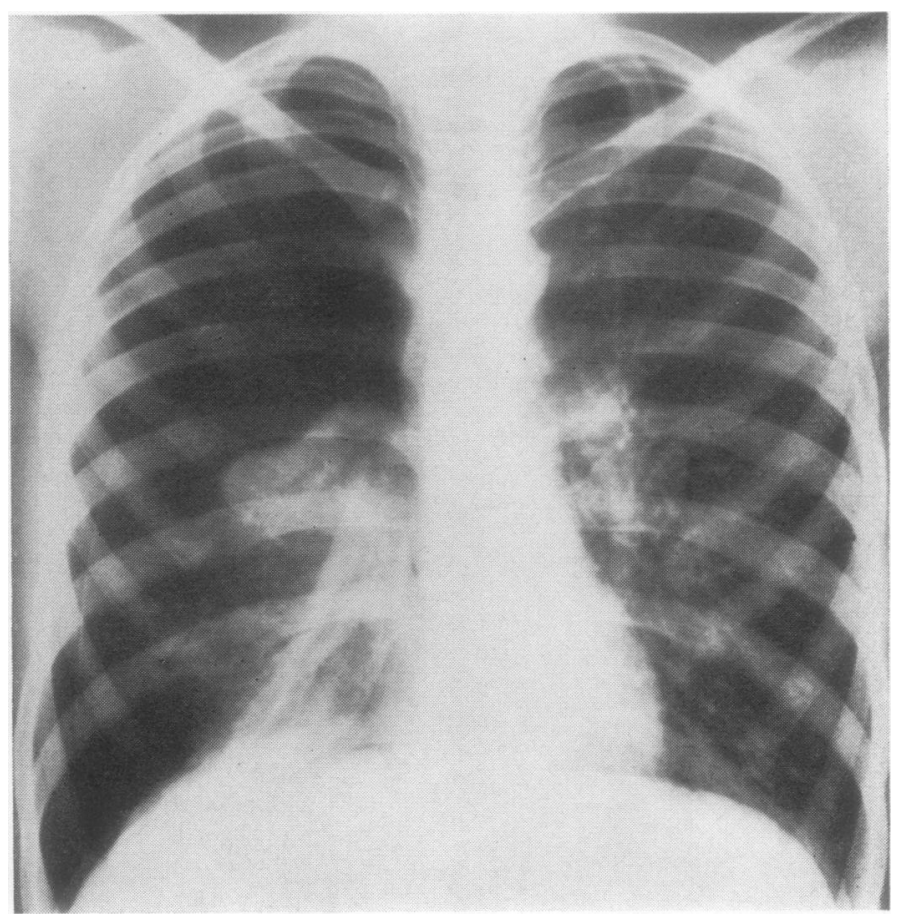




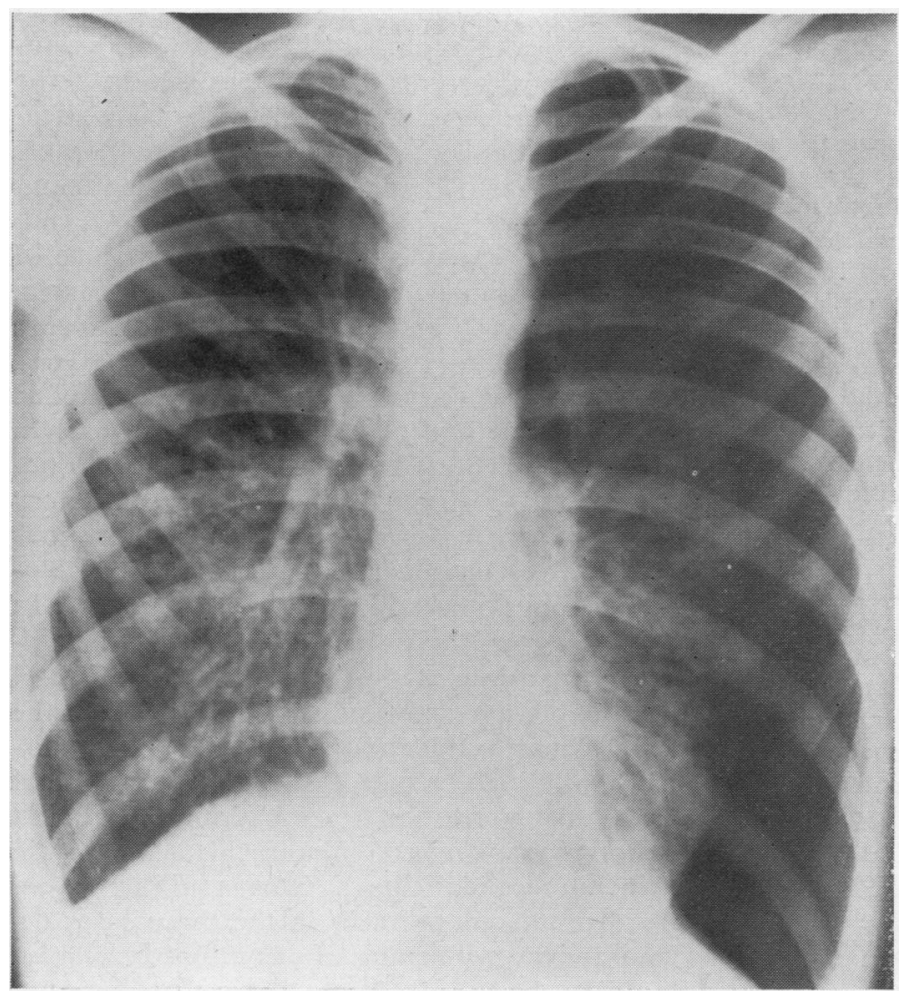

FIG. 6. Case 3. Chest radiograph (1.1.69) showing left pneumothorax.

FIG. 7. Case 3. Chest radiograph (24.1.69) after surgical management of the left-sided pneumothorax showing complete re-expansion of both lungs.

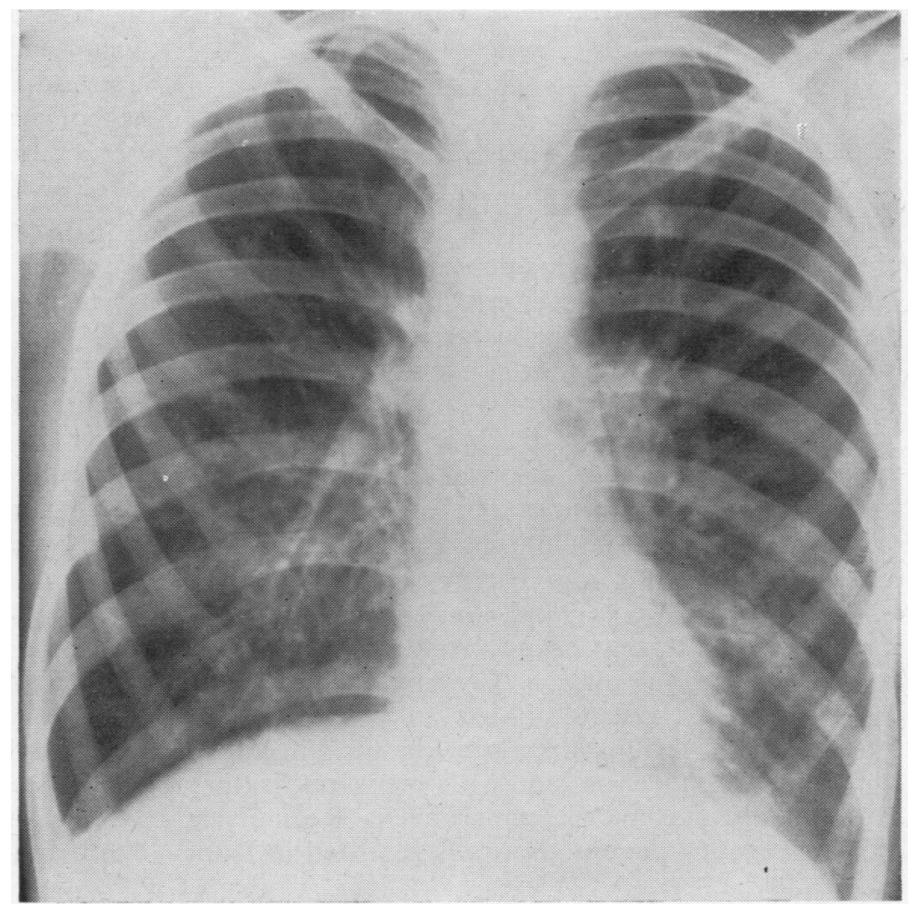


the right axilla. After 24 hours the right lung was fully expanded and the tube was clamped. However, on 4.5.68 there was a recurrence of the pneumothorax which did not respond to suction (Fig. 5), and on 15.5.68 the right parietal pleura was stripped from apex to hilum and down the aorta to the diaphragm. Post-operative recovery was uneventful. He remained well until 24.12.68, when he developed sudden left shoulder pain, and increasing shortness of breath during the following week. A chest radiograph on 1.1.69 confirmed a left spontaneous pneumothorax (Fig. 6). On 3.1.69 a left parietal pleurectomy was performed and the immediate post-operative period was uneventful. On 11.1.69, however, he developed some left-sided pleuritic pain, and an increase in size of the left pneumothorax was discovered: this pneumothorax had at no time been entirely eliminated. An intercostal tube was inserted and the left lung expanded quickly (Fig. 7). He was discharged from hospital very much improved but was readmitted two weeks later with an exacerbation of breathlessness associated with profuse purulent sputum. His chest radiograph showed a small left pneumothorax and an increase in patchy shadowing throughout all zones of both lungs. A left intercostal tube was inserted, but, despite intubation, aspiration of secretions and intermittent positive pressure respiration, he deteriorated and died with irreversible respiratory failure. At necropsy there was a small pneumothorax on the left side, though the lung was adherent at its apex and over the diaphragm. The right lung was adherent to the chest wall by filmy adhesions, and both lungs showed bronchopneumonic consolidation.

\section{DISCUSSION}

Spontaneous pneumothorax is a not infrequent complication of cystic fibrosis and in this series has occurred in 5 of 49 cases under observation, that is an approximate incidence of $10 \%$. In three cases pneumothorax developed bilaterally, in two of the patients simultaneously. It usually occurs several years after diagnosis, usually in late childhood or adolescence; the reason for this delay is unknown but it explains the high incidence in our patients, whose mean age was 22 years. While spontaneous pneumothorax in otherwise normal individuals is not usually hazardous, the prognosis of this complication in cystic fibrosis is unfavourable (di Sant 'Agnese and Andersen, 1959) and comparable with that found in older patients with severe airways obstruction (Leading article, 1968). A spontaneous pneumothorax in cystic fibrosis may cause a serious deterioration in ventilatory capacity, and in a patient with gross respiratory insufficiency may be a terminal event. Recurrence of spontaneous pneumothorax is reported in from
10 to $60 \%$ of otherwise normal individuals (Gobbel, Rhea, Nelson, and Daniel, 1963). Recurrence is almost certainly more frequent in cystic fibrosis, and in view of the hazards of complication and the danger of bilateral pneumothoraces pleurodesis has to be considered. Of the various methods available, parietal pleurectomy is undoubtedly associated with the lowest incidence of recurrence (Bromley, 1967 ; Heckscher, Larsen, and Lassen, 1966) and does not usually cause a restrictive ventilatory defect (Gobbel et al., 1963), so that provided the procedure is without undue risk it is the method of choice in this disease. In all three cases reported here there were severe obstructive and restrictive defects of ventilatory capacity, but there were no immediate and serious post-operative complications, probably because of energetic physiotherapy and chemotherapy. In case 3 , however, there was difficulty in maintaining the expansion of the left lung after surgery. This probably prevented subsequent binding of the visceral pleura to the chest wall before he was discharged, although he was well at this time, so that when he had a further exacerbation a small left pneumothorax returned, though this played little part in his terminal illness. Otherwise there were no further pneumothoraces in the three cases described and no worsening of symptoms or clinical state. It can be seen from the Table that there has been no gross deterioration in ventilatory capacity in cases 1 and 2 . In this context it is of interest to note the degree of deterioration in lung function induced by the small pneumothorax in case 1 , which developed two years or more after the pleurectomy on the opposite side.

Spontaneous pneumothorax as a late complication in cystic fibrosis is difficult to explain. If it were due to some mechanism similar to that which is responsible for pneumothorax in asthma or staphylococcal pneumonia it might be expected earlier in the course of the disease. Perhaps the pneumothoraces are associated with 'scar' emphysema (Laennec, 1819) or the abnormal tall columnar cells lining the pleura (Dunnill, 1959). Overdistension of alveoli following bronchial obstruction by mucus seems unlikely to account for the development of the pneumothoraces. Lifschitz et al. (1968) reported the 'common post-mortem findings' of bullae and subpleural blebs and the 'not uncommon' radiological identification of blebs and bullae in cystic fibrosis. This has not been our finding. In none of our patients was interstitial emphysema found. Blebs which are intra-pleural or intra-interstitial accumulations of air, as distinct from bullae which represent alveolar change, are 
a very uncommon finding in the normal or fibrocystic lung (Reid, 1967).

Any emphysema seen is not simply due to overdistension but is related to the alveolar hypoplasia or damage which is found in cystic fibrosis. A very irregular distribution of emphysema throughout the lung in cystic fibrosis has been noted (Davies, G. M., 1969, personal communication).

The possible significance of abnormal pleural cells found in cystic fibrosis must also be taken into account. Dunnill (1959) reported this metaplastic change over the whole visceral pleura of a patient with cystic fibrosis. It is not known at what age or stage of development these pleural changes first occur and whether they involve both visceral and parietal surfaces. A rupture of the abnormal pleura into the diseased alveolus or a spread of interstitial emphysema into the pleural space are two possible explanations. The onset of pneumothoraces in an earlier age group in cystic fibrosis than in the normal population is also more easily understood when the chronic and generalized nature of the pleural and alveolar abnormalities are considered together. In the case described by Dunnill some pleural adhesions were present, but they were extremely friable; indeed it is remarkable that the pleura so often remains free of adhesions despite the long-continued subpleural, intrapulmonary suppuration. This would suggest that the pleural surfaces of patients with cystic fibrosis are in some way unable to respond to the irritant effect of infection: they may similarly react poorly to the irritant effect of agents introduced into the pleural space in an attempt to promote adhesion between the pleural surfaces.

However, in view of the relative success of the three examples of pleurectomy reported here and the serious morbidity associated with the recurrence of a pneumothorax, we think that this treat- ment should be considered in the management of a spontaneous pneumothorax in a patient with cystic fibrosis.

We are indebted to Sir Kenneth Robson for case 3, to Professor Lynne Reid for her helpful advice, and to Mr. Matthias Paneth for carrying out the pleurectomies.

\section{REFERENCES}

Bromley, L. L. (1967). The management of spontaneous pneumothorax. Thorax, 22, 482.

di Sant 'Agnese, P. A., and Andersen, D. H. (1959). Cystic fibrosis of the pancreas in young adults. Ann. intern. Med., 50, 1321.

Dunnill, M. S. (1959). Metaplastic changes in the visceral pleura in a case of fibrocystic disease of the pancreas. J. Path. Bact., $77,299$.

Gobbel, W. G., Jr., Rhea, W. G., Jr., Nelson, I. A., and Daniel, R. A., Jr. (1963). Spontaneous pneumothorax. J. thorac. cardiovasc. Surg., 46, 331 .

Heckscher, Th., Larsen, O. A., and Lassen, N. A. (1966). A clinical method for determination of regional lung function using intravenous injection of $\mathrm{Xe}^{133}$. Scand. J. resp. Dis., Suppl. No. 62, p. 31 .

Ku lczycki, L. L., and LoPresti, J. M. (1967). Mucous retention and overinflation as basic pulmonary complications in cystic fibrosis (mucoviscidosis). Mod. Probl. Pädiat., 10, 207.

Laennec, R. T. H. (1819). De l'Auscultation Médiate, ou Traité du Diagnostic des Maladies des Poumons et du Coeur, vol. 1, p. 437, Brosson and Chaude, Paris.

Leading article (1968). Spontaneous pneumothorax. Brit. med. J., 1,720 .

Lifschitz, M. I., Bowman, F. O., Jr., Denning, C. R., and Wylie, R. H. (1968). Pneumothorax as a complication of cystic fibrosis. Amer. J. Dis. Child., 116, 633.

Reid, L. (1967). The Pathology of Emphysema. Lloyd Luke, London.

\section{ADDENDUM}

Since this paper was submitted, the authors have had two further patients with unilateral pneumothoraces. In one, a girl aged 15, the right-sided pneumothorax was transient, asymptomatic, and small. In the other, a man of 23 years, a left-sided pneumothorax required pleurectomy. This was carried out with no deterioration in ventilatory capacity. These two patients thus increase the observed incidence to approximately $14 \%$. 